\title{
ALMA Thermal Observations of Europa
}

\author{
Samantha K. Trumbo ${ }^{1}$ (D), Michael E. Brown ${ }^{1}(1)$, and Bryan J. Butler ${ }^{2}$ \\ ${ }^{1}$ Division of Geological and Planetary Sciences, California Institute of Technology, Pasadena, CA 91125, USA \\ ${ }^{2}$ National Radio Astronomy Observatory, Socorro, NM 87801, USA \\ Received 2018 March 21; revised 2018 August 9; accepted 2018 August 13; published 2018 September 18
}

\begin{abstract}
We present four daytime thermal images of Europa taken with the Atacama Large Millimeter Array. Together, these images comprise the first spatially resolved thermal data set with complete coverage of Europa's surface. The resulting brightness temperatures correspond to a frequency of $233 \mathrm{GHz}(1.3 \mathrm{~mm})$ and a typical linear resolution of roughly $200 \mathrm{~km}$. At this resolution, the images capture spatially localized thermal variations on the scale of geologic and compositional units. We use a global thermal model of Europa to simulate the ALMA observations in order to investigate the thermal structure visible in the data. Comparisons between the data and model images suggest that the large-scale daytime thermal structure on Europa largely results from bolometric albedo variations across the surface. Using bolometric albedos extrapolated from Voyager measurements, a homogenous model reproduces these patterns well, but localized discrepancies exist. These discrepancies can be largely explained by spatial inhomogeneity of the surface thermal properties. Thus, we use the four ALMA images to create maps of the surface thermal inertia and emissivity at our ALMA wavelength. From these maps, we identify a region of either particularly high thermal inertia or low emissivity near $90^{\circ}$ west and $23^{\circ}$ north, which appears anomalously cold in two of our images.
\end{abstract}

Key words: planets and satellites: general - planets and satellites: individual (Europa) - planets and satellites: surfaces

\section{Introduction}

Europa's icy surface is marked by fractured, ridged, and chaotic terrain suggestive of a history of geologic activity (e.g., Collins \& Nimmo 2009; Kattenhorn \& Hurford 2009; Prockter \& Patterson 2009). Spectroscopic studies have revealed multiple compositions that reflect the influences of both endogenous geologic processes (e.g., McCord et al. 1998; Fischer et al. 2015) and exogenous radiolytic processing by Jovian magnetospheric particles (e.g., Carlson et al. 1999, 2002), but the balance of these influences in shaping surface properties is not well understood. Surface temperature measurements can provide an additional window onto both types of processes. Such measurements present perhaps the best means for identifying regions of active geologic activity. Indeed, active hotspots persist at both the "tiger stripes" of Enceladus (Spencer et al. 2006) and the volcanoes of Io (Pearl \& Sinton 1982; Spencer et al. 1990). In addition, Cassini thermal observations of Saturn's moons Mimas and Tethys have shown that temperature measurements can reveal details on the effects of magnetospheric particle bombardment on surface texture (Howett et al. 2011, 2012). Finally, thermal data can provide insight on diurnal sublimation cycles, impact gardening by micrometeorites, and sputtering from particle impacts, which also affect the surface compositions and morphologies and, thus, the surface thermophysical properties.

To date, the only spatially resolved thermal data of Europa were collected by the Galileo Photopolarimeter-Radiometer (PPR). These data provided the first brightness temperature maps of the surface and included both daytime and nighttime measurements (Spencer et al. 1999). Modeling efforts using the PPR data have found thermal inertia values between 30 and $140 \mathrm{~J} /\left(\mathrm{m}^{2} \mathrm{~K} \mathrm{~s}^{1 / 2}\right)$, consistent with a particulate, uncompacted regolith texture unlike that of solid water ice (Spencer et al. 1999; Rathbun et al. 2010). However, the Galileo PPR only obtained limited coverage of the surface. Furthermore, from the end of the Galileo mission until very recently, subsequent brightness temperature measurements of similar quality and spatial resolution have been impossible to achieve. Recently, however, the Atacama Large Millimeter Array (ALMA) has made the collection of spatially resolved, highquality thermal data sets possible.

Here, we present four ALMA thermal images that together cover the entire surface of Europa at a frequency of $233 \mathrm{GHz}$ $(1.3 \mathrm{~mm})$ with a typical linear resolution of $\sim 200 \mathrm{~km}$. Using a global thermal model of Europa, we fit the observations and investigate the nature of thermal structure visible across the surface.

\section{ALMA Observations and Data Reduction}

The observations of Europa were taken with the main array of ALMA, which is composed of up to $5012 \mathrm{~m}$ diameter antennas spread across the Altiplano in the high northern Chilean Andes. Every pair of antennas acts as a two-element interferometer, and together these individual interferometers allow for the reconstruction of the the full sky brightness in both dimensions (Thompson et al. 2001).

ALMA can operate in seven frequency windows, from $\sim 90$ to $\sim 950 \mathrm{GHz}$. The observations presented here were taken in Band 6, near $230 \mathrm{GHz}$, in the "continuum" (or "TDM") mode, with the standard frequency tuning. This results in four spectral windows with frequencies: 223-225 GHz; $225-227 \mathrm{GHz}$; 239-241 GHz; and 241-243 GHz. In the data reduction, we average over the entire frequency range, and use $233 \mathrm{GHz}$ as the observation frequency in our thermal modeling.

We observed Europa with ALMA on 2015 November 17, 25,26 , and 27. For these observations, there were between 38 and 43 antennas, in the C36-7 configuration. This configuration 
Table 1

Table of Observations

\begin{tabular}{lcccc}
\hline \hline $\begin{array}{l}\text { Date } \\
\text { (UT) }\end{array}$ & $\begin{array}{c}\text { Time } \\
\text { (Start/End) }\end{array}$ & $\begin{array}{c}\text { Sub-Earth Longitude } \\
\text { Range }\end{array}$ & $\begin{array}{c}\text { Sub-Earth Latitude } \\
\text { Elliptical Beam Resolutions } \\
\text { (Milliarcseconds) }\end{array}$ & $\begin{array}{c}57 / 42 \\
2015 \text { Nov 17 }\end{array}$ \\
2015 Nov 25 & $14: 36 / 14: 50$ & $44.9-45.9$ & -1.44 & $59 / 53$ \\
2015 Nov 26 & $11: 25 / 11: 41$ & $121.5-122.6$ & -1.52 & $77 / 52$ \\
2015 Nov 27 & $08: 22 / 08: 38$ & $210-211.1$ & -1.52 & $54 / 53$ \\
\hline
\end{tabular}

has a maximum antenna spacing of $\sim 5 \mathrm{~km}$, giving a resolution on the sky of $\sim 50$ mas, and a minimum antenna spacing of $\sim 250 \mathrm{~m}$, giving a largest theoretical recoverable scale of $\sim 0.65$ arcsec. Details of observation times, geometries, and resolutions are given in Table 1.

All of these observations are in dual-linear polarization; in the end, we combine these into a measurement of Stokes I. While we expect polarized emission from the surface, it is relatively weak, and we did not measure the cross-polarized signals.

Each observation was about 20 minutes in duration, including all calibration overheads, which resulted in roughly 8 minutes on Europa. The point-like calibrator $\mathrm{J} 1058+0133$ was used as the absolute flux density scale calibrator for all four observations - it is part of a grid of calibrators, which are regularly monitored against the main flux density scale calibrators for ALMA (Butler 2012). The nearby calibrator $\mathrm{J} 1108+0811$ was used to calibrate the phase of the atmosphere and antennas as a function of time.

Initial calibration of the data was provided by the ALMA observatory and completed in the CASA reduction package (McMullin et al. 2007) via the ALMA pipeline (Muders et al. 2014). We exported the provided visibilities from CASA and continued the data reduction in the AIPS reduction package (Bridle \& Greisen 1994). We self-calibrated (Cornwell \& Fomalont 1999) the data in three iterations down to a time interval of 8 seconds (possible because Europa is such a bright target), using an initial limb-darkened model and imaging more deeply in each iteration (Butler \& Bastian 1999). The initial model in each iteration is parameterized to account for limb darkening and includes the diameter (taken to be known from the observing geometry), the total flux density, and and the limb-darkening parameter, where both the flux density and the limb-darkening parameter are taken from actual fits to the visibilities at each step using the AIPS task OMFIT.

Inspection of the resulting images from the four days of observation indicated that there was an apparent offset in the overall brightness of the November 17 data compared to the other days-the November 17 data was less bright by $\sim 10 \%$. Examination of the derived flux densities for $\mathrm{J} 1108+0811$ confirmed this offset. To determine the cause of the offset, we searched the ALMA grid calibrator monitoring catalog for flux densities derived for $\mathrm{J} 1058+0133$ (our flux density scale calibrator) over the period of time relevant to our observations and compared them to the flux densities used in the ALMA data reduction pipeline. We found that, while the cataloged flux densities at our frequency show the source decreasing in brightness from $3.42 \pm 0.07 \mathrm{Jy}$ on November 17 to $3.02 \pm 0.10 \mathrm{Jy}$ on November 25 , this decrease was not properly reflected in the values used in the data reduction pipeline (3.37 Jy for November 17 and $3.33 \mathrm{Jy}$ for November 25-27). We therefore made corrections to the flux density scale of the visibilities (and hence the brightness temperatures in the images) by accounting for the flux density differences between the measurements of $\mathrm{J} 1058+0133$ from November 17 and November 25 and the values used in the pipeline data reduction. The resultant correction factors are 1.015 for November 17 and 0.907 for the remaining three dates.

The final images are shown in Figure 1. They were produced using a robust parameter of zero, which is a good compromise between resolution and signal-to-noise. Pixel sizes are roughly a factor of 10 smaller than the actual resolution to minimize deconvolution errors. At the time of our observations, Europa's projected diameter was $\sim 0$ !" 77 on the sky, resulting in $\sim 15$ resolution elements across the disk. We note that this diameter is larger than the theoretical largest recoverable scale noted above. However, as the structure of the visibilities (and the sky brightness itself) is well-known for circular sources, and, as we use fits of the visibilities and an initial model incorporating the fitted zero-spacing flux density as the first step in all of our imaging, the overall brightness level is well-constrained (Butler \& Bastian 1999).

\section{Thermal Modeling}

We use a global thermal model of Europa, similar to those used for other solar system bodies (e.g., Spencer et al. 1989; Spencer 1990; Hayne \& Aharonson 2015), to simulate the ALMA observations. The model begins by calculating the absorbed solar flux at each point on the surface according to the local bolometric albedo. The resulting heat is then conducted through the near-surface layers, where the temperatures as a function of depth and time are controlled by the thermal inertia. The model numerically solves for these temperatures using a small global heat flux of $20 \mathrm{~mW} \mathrm{~m}^{-2}$ (Mitri \& Showman 2005; Barr \& Showman 2009) as a lower boundary condition and gray body radiation to space as an upper boundary condition. The end result is a radiative flux map of the surface that can be output for the geometries and times of the ALMA observations, smoothed to the ALMA resolution, and converted to brightness temperature. A complete description of the model can be found in Trumbo et al. (2017).

The primary difference in our modeling approach from those taken in the past for Europa (e.g., Spencer et al. 1999; Rathbun et al. 2010) is that we do not fit for bolometric albedo, but rather use an albedo map constructed from discrete Voyager measurements. We take the normal albedos for distinct locations on the surface from McEwen (1986) across the green, blue, violet, and ultraviolet Voyager filters. We then weight the values in each filter by the solar flux in the corresponding wavelength range and average the values. As the phase integral of Europa is 1.01 (Grundy et al. 2007), we take these averages as approximate bolometric albedos at each location. Finally, we take these as tie-points to the grayscale Voyager/Galileo global mosaic of Europa (USGS 2002). We 
Data
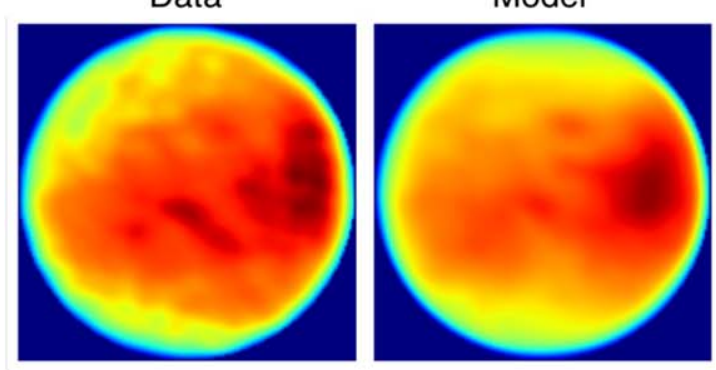

Sub-Jovian to Leading $\left(45^{\circ}\right)$
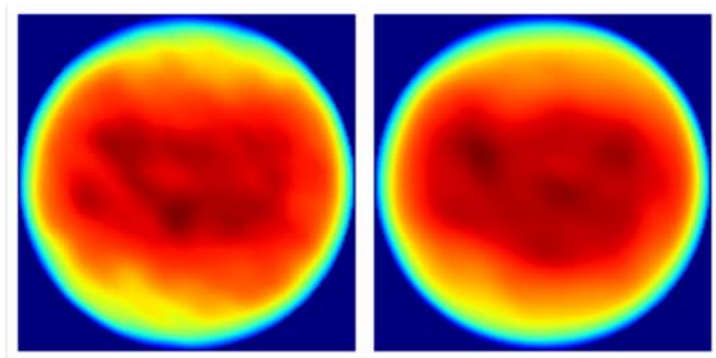

Anti-Jovian to Trailing $\left(210^{\circ}\right)$

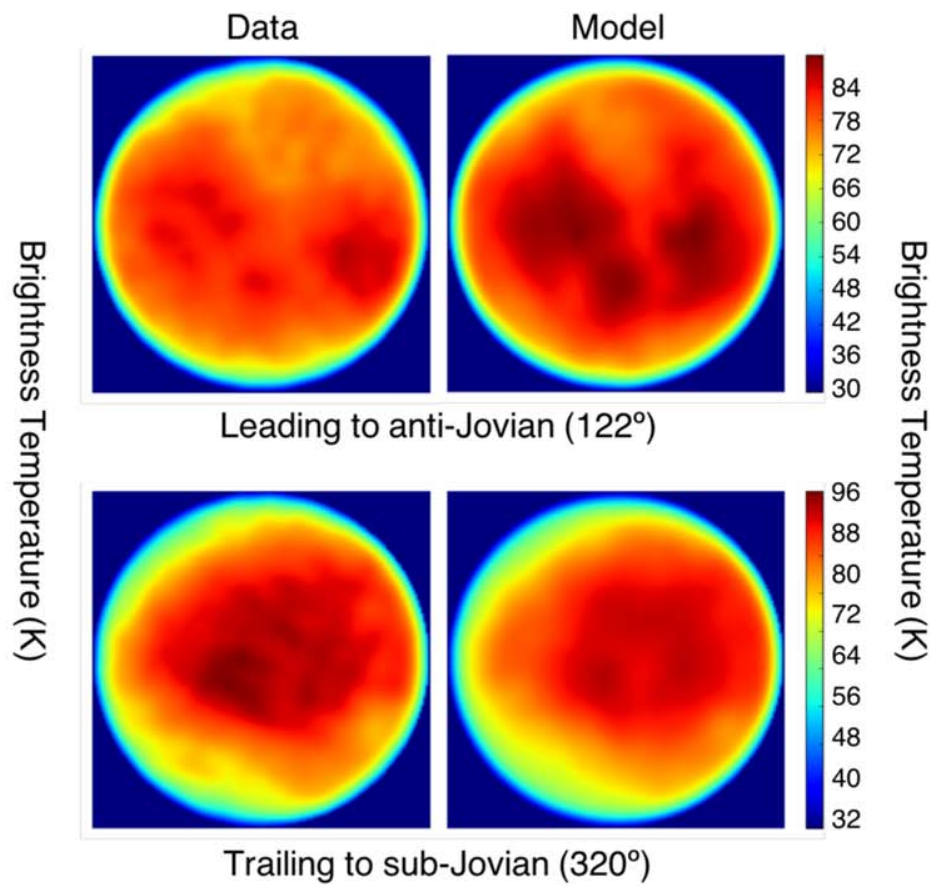

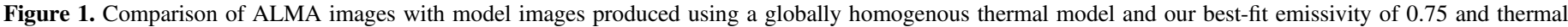
inertia of $95 \mathrm{~J} /\left(\mathrm{m}^{2} \mathrm{~K} \mathrm{~s}^{1 / 2}\right)$. Large-scale thermal structure is well-reproduced and many corresponding features are identifiable in each data-model pair.

fit a line to the relationship between the pixel values in the image at the Voyager albedo locations and the approximate bolometric albedo tie-points, and use this relationship to produce a high-resolution albedo map from the mosaic. The pixel values and albedo tie-points correlate with an $R^{2}$ of 0.92 and a standard deviation in albedo of 0.03 . We use the resulting map in our thermal modeling and treat the surface thermal inertia and emissivity at our ALMA wavelength as free parameters, assuming a fixed bolometric emissivity of 0.9 (Spencer 1987).

Bolometric emissivities less than one will increase the physical temperatures of the surface by inhibiting radiative heat loss. However, as ALMA observes brightness temperatures at $233 \mathrm{GHz}(1.3 \mathrm{~mm})$, rather than physical temperatures, a decrease in emissivity at ALMA wavelengths actually tends to decrease the observed brightness temperatures everywhere on the disk for the same reason. Variations in thermal inertia, on the other hand, affect temperatures differently depending on the time of day. An increase in thermal inertia will flatten the diurnal temperature curve by reducing the contrast between nighttime and midday temperatures. In contrast, a decrease in thermal inertia will steepen the diurnal temperature curve, lowering nighttime temperatures and increasing them at midday.

When fitting the data using our simple thermal model, however, the deduced thermal inertia and emissivity may also include minor contributions from physical processes not included in the model. In particular, our model does not include sunlight penetration with depth in the regolith, thermal emission from depth in the regolith, or surface roughness. As described in Trumbo et al. (2017), our model assumes that the solar flux is absorbed in the topmost model layer, which is a standard assumption for several thermal models (e.g., Spencer et al. 1989; Spencer 1990; Hayne \& Aharonson 2015) and particularly valid for low-albedo surfaces. However, sunlight may penetrate deeper into a relatively high-albedo particulate surface like that of Europa (Brown \& Matson 1987; Urquhart \& Jakosky 1996), resulting in heat at depth in the regolith. In practice, this effect is difficult to distinguish from that of thermal inertia (Urquhart \& Jakosky 1996); thus, we include only thermal inertia in our model.

Our model also assumes that the thermal emission detected by ALMA originates in the topmost model layer. At a frequency of $233 \mathrm{GHz}(1.3 \mathrm{~mm})$, however, ALMA is likely sensing slightly deeper into the surface. Thus, modeled brightness temperatures for a fixed emissivity and thermal inertia are slightly higher than they would be if this effect were included. We find that the inclusion of sunlight absorption at depth (at an e-folding of $2 \mathrm{~cm}$ ) and thermal emission from depth (with an e-folding of $1 \mathrm{~cm}$ ) results in slight changes to the model thermal inertia and emissivity, respectively, but has little effect on our conclusions from the data. Similarly, the inclusion of surface roughness appears to have little effect on our results. Surface roughness tends to increase surface temperatures, particularly at the limbs. However, using a similar implementation of surface roughness to Hayne \& Aharonson (2015), we find that rms slopes up to $20^{\circ}$ have relatively minor effects on our model fits to the data.

In our analysis, we aim to explain the nature of the largescale thermal structure of Europa and search for any potential systematic variation of thermal properties across the surface, rather than precisely determine the true values of the individual surface thermal properties. Thus, we use our simplest model and note that our model parameters may reflect the influences of the above effects.

\section{Fits to ALMA Observations}

We begin our analysis by attempting to determine a global best-fit emissivity and thermal inertia. We simulate the four ALMA observations over a grid of thermal inertias and emissivities and find the single best-fit values to these data by 


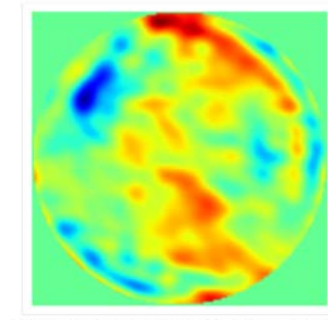

Sub-Jovian to Leading $\left(45^{\circ}\right)$

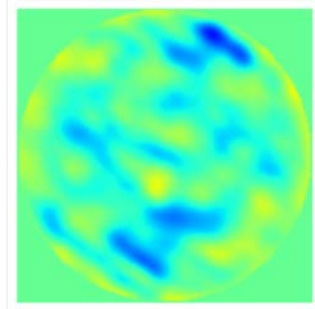

Anti-Jovian to Trailing $\left(210^{\circ}\right)$ Trailing to sub-Jovian $\left(320^{\circ}\right)$

Figure 2. Residuals between the data and model images produced with our best-fit parameters, where positive temperatures indicate places where the data are hotter than the model predicts. Discrepancies may result from local variations in the surface thermal properties.

minimizing the square of the residuals between model and data images. This initial fitting produces a global best-fit emissivity of 0.75 and a best-fit thermal inertia of $95 \mathrm{~J} /\left(\mathrm{m}^{2} \mathrm{~K} \mathrm{~s}^{1 / 2}\right)$. This thermal inertia is higher than the value of $70 \mathrm{~J} /\left(\mathrm{m}^{2} \mathrm{~K} \mathrm{~s}^{1 / 2}\right)$ found by Spencer et al. (1999) for the equatorial regions, but lies within the range of $30-140 \mathrm{~J} /\left(\mathrm{m}^{2} \mathrm{~K} \mathrm{~s}^{1 / 2}\right)$ mapped by Rathbun et al. (2010).

Data images are shown in Figure 1 alongside model images produced using these global best-fit parameters. The globally homogenous model, where only albedo varies spatially across the surface, reproduces the large-scale thermal structure of the data well. This suggests that the majority of the visible daytime thermal structure on Europa is governed primarily by local albedo variations, rather than variations in internal heating, thermal inertia, or emissivity. However, when we difference the model and data images, localized discrepancies between the data and model do become apparent. Figure 2 shows the residuals between each data-model image pair from Figure 1, where positive temperatures indicate regions where the data are hotter than the model predicts. Such discrepancies are not necessarily surprising given the inhomogeneous nature of Europa's surface, and we do not expect the entire surface, with its varying compositions and morphologies, to be wellrepresented by a single thermal inertia or emissivity.

In principle, discrepancies may be due to a number of possible causes. Localized differences in the thermal inertia, emissivity, or albedo from the values used in our model will result in residuals on the spatial scale of the geographic variability in these properties. Other possible contributing factors include spatial variation in the transparency of the surface to sunlight or thermal emission, which may manifest as apparent thermal inertia or emissivity discrepancies in our modeling. As we lack observations of the same regions at multiple times of day over most of the surface, we cannot conclusively distinguish between these potential causes everywhere. For instance, most of the positive temperature anomalies in the sub-Jovian to leading hemisphere image of Figure 2 can be equivalently explained by a decrease in albedo of $\sim 8-20 \%$ or an elevated subsurface heat flux of $\sim 1.2-2 \mathrm{~W} \mathrm{~m}^{-2}$ (consistent with liquid water a few hundred meters below the surface). However, as we provide the model with spatially varying albedos derived from concrete measurements and because other objects in the solar system exhibit significant localized differences in thermal inertia (e.g., Putzig et al. 2005; Howett et al. 2010, 2011, 2012; Hayne et al. 2017), we experiment with the idea that the residuals may be caused by thermal inertia variations.

We fix the emissivity at the global best-fit value of 0.75 and fit the data by letting the thermal inertia vary at each point. Points near the limbs of each observation are foreshortened, convolved with the cold sky, and more sensitive to surface roughness, positioning uncertainty, and other effects that are unimportant away from the limb. In addition, these locations are experiencing times of day near where diurnal curves for varying thermal inertias (at a given emissivity) intersect. The combination of this effect with the magnified uncertainties near the limbs can result in widely varying best-fit thermal inertias that are discontinuous with those of surrounding areas. Thus, we only fit data within $57^{\circ}$ of the central point of each observation, which we find minimizes this effect without compromising our longitudinal coverage. In fitting regions that appear in two overlapping images, we use both times of day in the fitting. This produces a map of thermal inertia of the surface (Figure 3), assuming a globally homogenous emissivity and our bolometric albedo map constructed from Voyager measurements.

Under the assumption that all of the residuals between the ALMA data and the global best-fit model can be attributed to thermal inertia variations, typical thermal inertias on Europa range between 40 and $300 \mathrm{~J} /\left(\mathrm{m}^{2} \mathrm{~K} \mathrm{~s}^{1 / 2}\right)$, with the lowest average values on the sub-Jovian hemisphere and the highest average values between the leading and anti-Jovian hemispheres. However, it is important to note that some patterns interpreted to be thermal inertia in this map may, in reality, be due to patterns in emissivity or albedo. In fact, this method does not completely eliminate residuals in all regions of overlap between the images, and minor discrepancies (primarily $\leqslant 3 \mathrm{~K}$ ) remain, implying some underlying differences in emissivity or albedo from those values used.

We extrapolate our albedos from relatively few Voyager measurements, and this extrapolation may not work equally well for all locations on the surface. As mentioned above, the scatter between our best-fit albedo model and the measured albedo tie-points is $+/-0.03$, which results in adjustments to the best-fit local thermal inertia between $10 \%$ and $60 \%$ for most locations on the surface. However, we do not see any obvious correlation between residuals, derived thermal inertias, and albedo. Furthermore, this map does produce a locally elevated thermal inertia surrounding the crater Pwyll, which is consistent with nighttime PPR data of the same region (Spencer et al. 1999; Trumbo et al. 2017) and the tendency of crater ejecta to exhibit elevated thermal inertia elsewhere in the solar system (e.g., Mellon et al. 2000; Hayne et al. 2017).

However, we can take a complementary approach and assume that the residuals of Figure 2 are instead due to variations in the surface emissivity at our ALMA wavelength of $1.3 \mathrm{~mm}$. Fixing the thermal inertia at our global best-fit value of $95 \mathrm{~J} /\left(\mathrm{m}^{2} \mathrm{~K} \mathrm{~s}^{1 / 2}\right)$ and letting the emissivity vary at each point produces a similar map of emissivity at $1.3 \mathrm{~mm}$ (Figure 3 ) that alternatively fits the data with residuals of comparable 


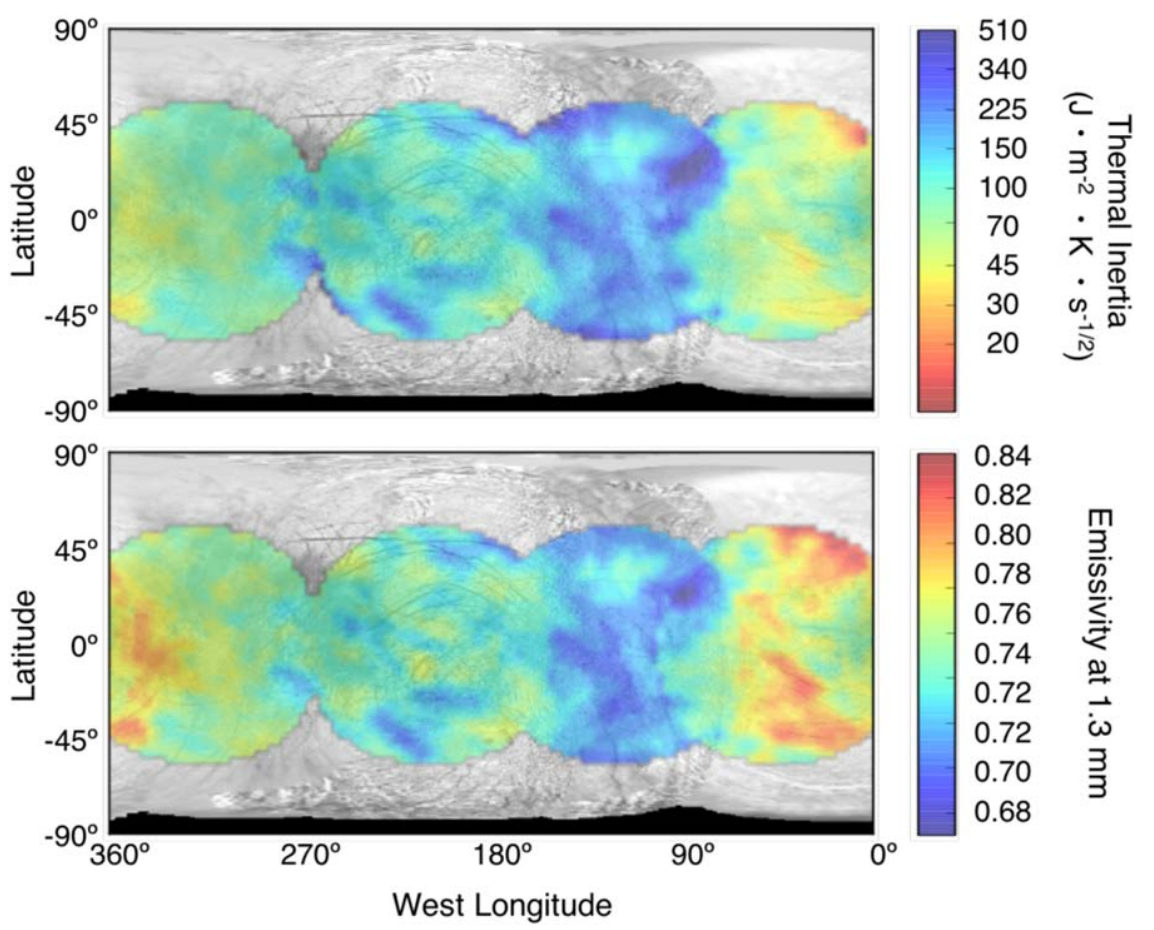

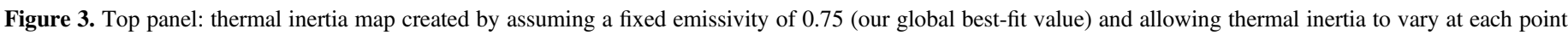

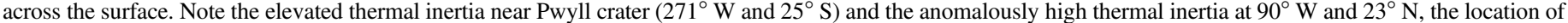

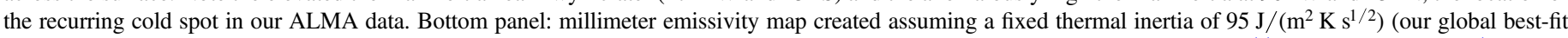
value) and allowing emissivity to vary spatially across the surface. The background basemap is from USGS Map-a-Planet: https://www.mapaplanet.org/.

magnitude in areas of overlap. Thus, the residuals of Figure 2 likely represent a combination of thermal inertia and emissivity variations. Indeed, we find both the fitted thermal inertia and emissivity ranges to be physically plausible. All derived thermal inertias are less than that of solid water ice, and the low end of our fitted thermal inertia range is consistent with thermal inertias derived for the Saturnian satellites (Howett et al. 2010). Similarly, the depressed millimeter emissivities we derive are consistent with those derived for Kuiper Belt and trans-Neptunian objects (Brown \& Butler 2017; Lellouch et al. 2017).

The most striking feature of both maps is the localized region of either highly elevated thermal inertia or low emissivity (deep blue in Figure 3 ) near $90^{\circ} \mathrm{W}$ and $23^{\circ} \mathrm{N}$, which coincides with the coldest spot in both the sub-Jovian to leading hemisphere and leading to anti-Jovian hemisphere images in Figure 2. The fact that we see this anomaly twice in two different images and at two different times of day strongly suggests that it corresponds to a region that is truly distinct in its thermal properties. While the maps suggest that the anomaly could either be explained by an elevated thermal inertia or a low emissivity, the morning temperature measurement at this location is too cold to be fit satisfactorily by thermal inertia alone, and both measurements are best fit by a moderate thermal inertia of $80 \mathrm{~J} /\left(\mathrm{m}^{2} \mathrm{~K} \mathrm{~s}^{1 / 2}\right)$ and a low emissivity of 0.66. A locally high thermal inertia may indicate a region of larger average particle size or of greater transparency to sunlight. A locally depressed emissivity may also be related to particle size (and therefore subsurface scattering properties) or may indicate a compositional difference resulting in a distinct spectral emissivity or more transparent surface at the ALMA wavelength (e.g., Lellouch et al. 2016, 2017).
This region coincides with the location of highest water-ice abundance mapped by Brown \& Hand (2013) and the location suggested to have the most crystalline water ice by Ligier et al. (2016). However, it does not correspond to any particular geologic unit (Leonard et al. 2017) or any unusual visible morphological or albedo features (USGS 2002). The anomaly is located within a region of relatively low-resolution imaging and was not covered by the published Galileo PPR data, so it is possible that a corresponding morphological, geologic, or thermal feature was simply not seen by Galileo. For instance, one might imagine that recent resurfacing, perhaps via meltthrough or diapirism, both of which are proposed explanations for nearby Murias Chaos at $84^{\circ} \mathrm{W}$ and $22^{\circ} \mathrm{N}$ (Figueredo et al. 2002; Fagents 2003), could have resulted in a region of recently extruded material with corresponding morphological expressions and different thermal properties than the surrounding regolith. It is also interesting to note that the anomaly is almost directly antipodal to Pwyll, the largest fresh crater on Europa at $271^{\circ} \mathrm{W}$ and $25^{\circ} \mathrm{S}$. Antipodal focusing of impact ejecta has been suggested as a potential explanation for a high thermal inertia deposit and corresponding geologic features on the Moon that are approximately antipodal to Tycho crater (Bandfield et al. 2017; Hayne et al. 2017). However, Tycho was likely the result of a more powerful impact and is roughly three times larger than Pwyll in diameter. Unfortunately, without new, high-resolution imaging, the potential association of this feature with unique geology will likely remain an open question.

Curiously, with the exception of Pwyll crater, our analysis has not identified any clear correlations between the potential thermal inertia values and geologic or compositional units. Although, this is not necessarily surprising, if one draws analogy to the Moon, where thermal inertia reliably follows 
impacts, but not major geologic units (Hayne et al. 2017). Furthermore, unlike Mimas and Tethys (Howett et al. 2011, 2012), and despite its location within Jupiter's magnetosphere, Europa also appears to carry no obvious thermal inertia signature of high-energy electron bombardment. On Mimas and Tethys, the thermal inertia anomalies are associated with the leading hemisphere bombardment of electrons with energies $\geqslant 1 \mathrm{MeV}$ (the energy needed for movement against the co-rotation direction of Saturn's magnetosphere) (Howett et al. 2011, 2012). It is possible that Europa lacks such a signature because the energy threshold for movement against co-rotation is much higher in Jupiter's magnetosphere ( $\sim 25 \mathrm{MeV}$ ) (Paranicas et al. 2007, 2009), so electrons with energies $>1 \mathrm{MeV}$ bombard both the leading and trailing hemispheres of Europa.

Ideally, we would include Galileo PPR data in this analysis. However, while our modeling approach reproduces the ALMA images quite well, it does not produce the same quality fits to the Galileo daytime data. When we try to incorporate the Galileo daytime data into our analysis, we find that the Galileo and ALMA data seem to prefer drastically different thermal properties, even in areas of overlap, such that the inclusion of the Galileo PPR data leaves residuals that appear systematic. In fact, when we attempt to model the Galileo PPR data alone, as we have done with the ALMA data set, we still obtain systematic residuals, which are only eliminated if we allow albedo and thermal inertia to vary simultaneously. This approach, however, requires bolometric albedo patterns over much of the surface that show little correspondence with the Voyager/Galileo mosaic or our ALMA images. We suspect this may be the result of unexplained systematics in the PPR data, rather than real properties of Europa's surface. As we are unable to explain why the daytime PPR data is inconsistent with the ALMA daytime data in areas of overlap, we opt to forego using any PPR data and instead focus on our selfconsistent ALMA data set. Despite this, our analysis does reproduce the high thermal inertia near Pwyll crater that was derived using both ALMA data and Galileo PPR nighttime data (Rathbun \& Spencer 2017; Trumbo et al. 2017). If we apply the same minor albedo adjustment near Pwyll as Trumbo et al. (2017), which is within our albedo errors, we arrive at a similar thermal inertia using only the ALMA data.

\section{Conclusions}

We obtained four ALMA thermal observations of Europa, which together cover the entire surface and reveal significant thermal structure. Using a globally homogenous, one-dimensional thermal model and a bolometric albedo map constructed from Voyager measurements, we are able to reproduce much of this structure well, indicating that it is primarily a product of bolometric albedo variation across the surface and the passive absorption and re-emission of sunlight. However, despite the similarity of the data and model images, there are localized disagreements, which may indicate variability in the surface thermophysical properties. We examine the possibility that these discrepancies can be explained by local thermal inertia variations and construct a corresponding thermal inertia map, assuming a globally homogenous millimeter emissivity. The map suggests typical values of the surface thermal inertia ranging from 40 to $300 \mathrm{~J} /\left(\mathrm{m}^{2} \mathrm{~K} \mathrm{~s}^{1 / 2}\right)$, with the lowest thermal inertias on the sub-Jovian hemisphere and the highest between the leading and anti-Jovian hemispheres. We also construct a complementary map of emissivity at our ALMA wavelength $(1.3 \mathrm{~mm})$, assuming a globally homogenous thermal inertia, which suggests emissivities of $0.67-0.84$. We find little correlation of thermal properties with geology or composition and few noteworthy anomalies, with the exception of an elevated thermal inertia surrounding Pwyll crater and a region of low emissivity or extremely elevated thermal inertia near $90^{\circ}$ $\mathrm{W}$ and $23^{\circ} \mathrm{N}$ on the leading hemisphere, in a region of relatively low-quality Galileo imaging. This leading hemisphere location corresponds to the region spectroscopically determined to be the iciest (and potentially most crystalline) on the surface. However, it does not coincide with any unique geologic or morphological features, nor was it covered by the Galileo PPR. Thus, while we suggest that the area is distinct in its thermal properties, we can only speculate as to its origins. Future ALMA observations will provide measurements of each location on the surface at other times of day, which will allow for better constraints on the surface thermal properties and, thus, their potential influences.

This paper makes use of the following ALMA data: ADS/ JAO.ALMA\#2015.1.01302.S. ALMA is a partnership of ESO (representing its member states), NSF (USA) and NINS (Japan), together with NRC (Canada), MOST and ASIAA (Taiwan), and KASI (Republic of Korea), in cooperation with the Republic of Chile. The Joint ALMA Observatory is operated by ESO, AUI/NRAO and NAOJ. The National Radio Astronomy Observatory is a facility of the National Science Foundation operated under cooperative agreement by Associated Universities, Inc. This research was supported by grant 1313461 from the National Science Foundation, as well as by a NASA Earth and Space Science Fellowship. The authors thank John R. Spencer, Julie A. Rathbun, James T. Keane, and Katherine R. de Kleer for useful conversations. The authors would also like to thank the anonymous referee, whose insightful comments led to the improvement of this manuscript.

Software: ALMA pipeline (Muders et al. 2014), CASA (McMullin et al. 2007), AIPS (Bridle \& Greisen 1994).

\section{ORCID iDs}

Samantha K. Trumbo (iD https://orcid.org/0000-00020767-8901

Michael E. Brown (iD https://orcid.org/0000-0002-8255-0545

\section{References}

Bandfield, J. L., Cahill, J. T. S., Carter, L. M., et al. 2017, Icar, 283, 282 Barr, A. C., \& Showman, A. P. 2009, in Europa, ed. R. T. Pappalardo, W. B. McKinnon, \& K. Khurana (Tucson, AZ: Univ. Arizona Press), 405

Bridle, A. H., \& Greisen, E. W. 1994, NRAO Memo, https://library.nrao.edu/ public/memos/aips/memos/AIPSM_087.pdf

Brown, M. E., \& Butler, B. J. 2017, AJ, 154, 19

Brown, M. E., \& Hand, K. P. 2013, AJ, 145, 110

Brown, R. H., \& Matson, D. L. 1987, Icar, 72, 84

Butler, B., \& Bastian, T. S. 1999, in ASP Conf. Ser. 180, Synthesis Imaging in Radio Astronomy II, ed. G. B. Taylor, C. L. Carilli, \& R. A. Perley (San Francisco, CA: ASP), 625

Butler, B. J. 2012, ALMA Memo, 594

Carlson, R. W., Anderson, M. S., Johnson, R. E., Schulman, M. B., \& Yavrouian, A. H. 2002, Icar, 157, 456

Carlson, R. W., Johnson, R. E., \& Anderson, M. S. 1999, Sci, 286, 97

Collins, G., \& Nimmo, F. 2009, in Europa, ed. R. T. Pappalardo, W. B. McKinnon, \& K. Khurana (Tucson, AZ: Univ. Arizona Press), 259

Cornwell, T., \& Fomalont, E. B. 1999, in ASP Conf. Ser. 180, Synthesis Imaging in Radio Astronomy II, ed. G. B. Taylor, C. L. Carilli, \& R. A. Perley (San Francisco, CA: ASP), 187 
Fagents, S. A. 2003, JGRE, 108, 5139

Figueredo, P. H., Chuang, F. C., Rathbun, J. A., Kirk, R. L., \& Greeley, R. 2002, JGRE, 107, 5026

Fischer, P. D., Brown, M. E., \& Hand, K. P. 2015, AJ, 150, 164

Grundy, W. M., Buratti, B. J., Cheng, A. F., et al. 2007, Sci, 318, 234

Hayne, P. O., \& Aharonson, O. 2015, JGRE, 120, 1567

Hayne, P. O., Bandfield, J. L., Siegler, M. A., et al. 2017, JGRE, 122, 2371

Howett, C. J. A., Spencer, J. R., Hurford, T., Verbiscer, A., \& Segura, M. 2012, Icar, 221, 1084

Howett, C. J. A., Spencer, J. R., Pearl, J., \& Segura, M. 2010, Icar, 206, 573

Howett, C. J. A., Spencer, J. R., Schenk, P., et al. 2011, Icar, 216, 221

Kattenhorn, S. A., \& Hurford, T. 2009, in Europa, ed. R. T. Pappalardo, W. B. McKinnon, \& K. Khurana (Tucson, AZ: Univ. Arizona Press), 199

Lellouch, E., Moreno, R., Müller, T., et al. 2017, A\&A, 608, A45

Lellouch, E., Santos-Sanz, P., Fornasier, S., et al. 2016, A\&A, 588, A2

Leonard, E. J., Patthoff, D. A., Senske, D. A., et al. 2017, LPSC, 48, 2357

Ligier, N., Poulet, F., Carter, J., Brunetto, R., \& Gourgeot, F. 2016, AJ, 151,163

McCord, T. B., Hansen, G. B., Clark, R. N., et al. 1998, JGRE, 103, 8603

McEwen, A. S. 1986, JGRB, 91, 8077

McMullin, J. P., Waters, B., Schiebel, D., Young, W., \& Golap, K. 2007, in ASP Conf. Ser. 376, Astronomical Data Analysis Software and Systems XVI., ed. R. A. Shaw, F. Hill, \& D. J. Bell (San Francisco, CA: ASP), 127 Mellon, M. T., Jakosky, B. M., Kieffer, H. H., \& Christensen, P. R. 2000, Icar, 148,437

Mitri, G., \& Showman, A. P. 2005, Icar, 177, 447

Muders, D., Wyrowski, F., Lightfoot, J., et al. 2014, in ASP Conf. Ser. 485, Astronomical Data Analysis Software and Systems XXIII., ed. N. Manset \& P. Forshay (San Francisco, CA: ASP), 383
Paranicas, C., Cooper, J. F., Garrett, H. B., Johnson, R. E., \& Sturner, S. J. 2009, in Europa, ed. R. T. Pappalardo, W. B. McKinnon, \& K. Khurana (Tucson, AZ: Univ. Arizona Press), 529

Paranicas, C., Mauk, B. H., Khurana, K., et al. 2007, GeoRL, 34, 15103

Pearl, J. C., \& Sinton, W. M. 1982, in Satellites of Jupiter (Tucson, AZ: Univ. Arizona Press), 724

Prockter, L. M., \& Patterson, G. W. 2009, in Europa, ed. R. T. Pappalardo, W. B. McKinnon, \& K. Khurana (Tucson, AZ: Univ. Arizona Press)

Putzig, N. E., Mellon, M. T., Kretke, K. A., \& Arvidson, R. E. 2005, Icar, 173,325

Rathbun, J. A., Rodriguez, N. J., \& Spencer, J. R. 2010, Icar, 210, 763

Rathbun, J. A., \& Spencer, J. R. 2017, in AGU Fall Meeting Abstracts (Washington, D.C.: American Geophysical Union), P52B-07

Spencer, J. R. 1987, PhD thesis, Univ. Arizona

Spencer, J. R. 1990, Icar, 83, 27

Spencer, J. R., Lebofsky, L. A., \& Sykes, M. V. 1989, Icar, 78, 337

Spencer, J. R., Pearl, J. C., Segura, M., et al. 2006, Sci, 311, 1401

Spencer, J. R., Shure, M. A., Ressler, M. E., et al. 1990, Natur, 348, 618

Spencer, J. R., Tamppari, L. K., Martin, T. Z., \& Travis, L. D. 1999, Sci, 284, 1514

Thompson, A. R., Moran, J. M., \& Swenson, G. W. 2001, Interferometry and Synthesis in Radio Astronomy (2nd ed.; New York: Wiley-Interscience)

Trumbo, S. K., Brown, M. E., \& Butler, B. J. 2017, AJ, 154, 148

Urquhart, M. L., \& Jakosky, B. M. 1996, JGRE, 101, 21169

USGS 2002, Controlled photomosaic map of Europa, Je 15M CMN: U.S. Geological Survey Investigations Series I-2757 